\title{
Arch replacement following endovascular arch repair for an infected stent: case report
}

\author{
Ana Lopez-Marco, Saleem Jahangeer, Benjamin Adams, Aung Ye Oo \\ Department of Cardiothoracic Surgery, St Bartholomew's Hospital, London, UK \\ Correspondence to: Miss Ana Lopez-Marco. Department of Cardiothoracic Surgery, Barts Heart Centre, St Bartholomew's Hospital, West Smithfield, \\ EC1A 7BE, London, UK. Email: ana.lopez-marco@nhs.net.
}

\begin{abstract}
Endovascular graft infection is a rare but challenging complication that requires a tailored approach to remove the infected graft and restore the arterial circulation combined with long-term antibiotic therapy. We present a case with a previous endovascular repair of the aortic arch and descending thoracic aorta on a 78-year-old male deemed too high risk for surgery. Three years after the endovascular repair, he presented with fever of unknown origin and the diagnosis of stent graft infection was made based on clinical, microbiological and radiological criteria. The peri-graft infection within the arch had dislocated the stent branch on the innominate artery and caused and expanding aneurysm. Urgent surgical indication was made based on the radiological findings and he underwent a removal of the infected material followed by aortic arch and descending aorta reconstruction with a total arch replacement with a frozen elephant trunk device using cardiopulmonary bypass with moderate hypothermic circulatory arrest and antegrade cerebral perfusion. The patient made an uneventful recovery and is free of recurrence eighteen months after the operation. Treatment of endovascular graft infections require a tailored treatment after multidisciplinary team discussions. In this particular case, the patient was deemed too high risk for surgery in the first instance, but we demonstrated that with treated in highly specialised unit, uneventful recovery is possible even after a more complex aortic repair following the endovascular infection. Complex aortic repair benefits always from a specialised multi-disciplinary team discussion and referral to high volume centers in order to achieve best results.
\end{abstract}

Keywords: Aortic arch; descending thoracic aorta; endovascular repair; graft infection; case report

Received: 11 April 2020; Accepted: 27 July 2020; Published: 20 January 2022.

doi: 10.21037 /jovs-20-100

View this article at: http://dx.doi.org/10.21037/jovs-20-100

\section{Introduction}

Endovascular graft infection is a rare but challenging complication, with a complex diagnosis and no standard treatment. A tailored approach should always be offered with the ultimate goal being to remove the infected graft which is not always feasible due to general fitness or comorbidities. A multidisciplinary team (MDT) discussion is warranted to find a procedure that combines the best morbidity, mortality and durability results. We present the following case in accordance with the CARE reporting checklist (available at https://jovs.amegroups.com/article/ view/10.21037/jovs-20-100/rc).

\section{Case presentation}

A 78-year-old male with history a previous total arch endovascular repair of an extensive aneurysm of the distal arch and descending thoracic aorta using a branched arch device into the innominate and left common carotid arteries (Zenith Arch Endograft ${ }^{\mathrm{TM}}$ and Zenith Alpha ${ }^{\mathrm{TM}}$ endovascular distal extension; Cook Medical, Bloomington, USA) and a prior left common carotid to left subclavian bypass 3 years ago, was referred to our department with a confirmed infection of the endovascular graft for consideration of urgent surgery.

He presented with persistent pyrexia of unknown origin and the diagnosis of the graft infection was made base on 


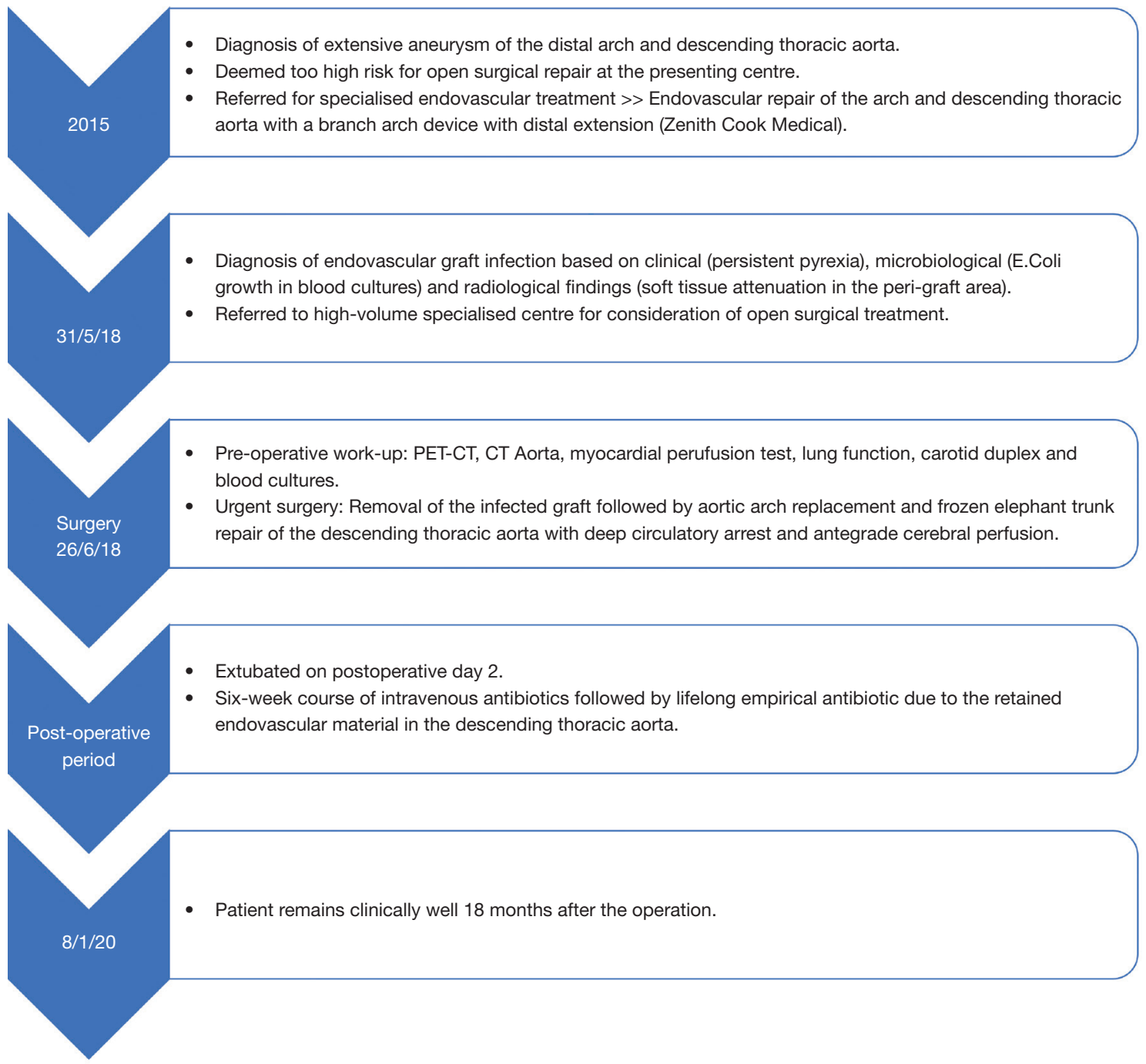

Figure 1 Timeline sequence of events since initial diagnosis of the extensive aortic aneurysm.

clinical, microbiological and radiological features. The original blood cultures revealed a growth of Escherichia coli, and he was treated according to sensitivity with a combined regime of Meropenem and Vancomycin (Figure 1).

Other comorbidities included hypertension, hypercholesterolaemia, remote smoking history and a previous open infrarenal aneurysm repair.

Written informed consent was obtained from the patient for video recording and use of the anonymised material for teaching and research purposes. All procedures performed in this study were in accordance with the ethical standards of the institutional and/or national research committee(s) and with the Helsinki
Declaration (as revised in 2013).

\section{Patient work-up}

As part of the preoperative work-up we requested a positron emission tomography-CT (PET-CT) that confirmed infection of the endovascular graft by demonstrating intense avidity in the innominate artery and distal aortic arch (Video 1).

An updated contrasted CT Aorta showed expansion of the aneurysm at the origin of the innominate artery with dislocation of the stent branch, as well as radiological signs of infection within the distal arch / proximal descending aorta (soft-tissue attenuation) and the large residual 


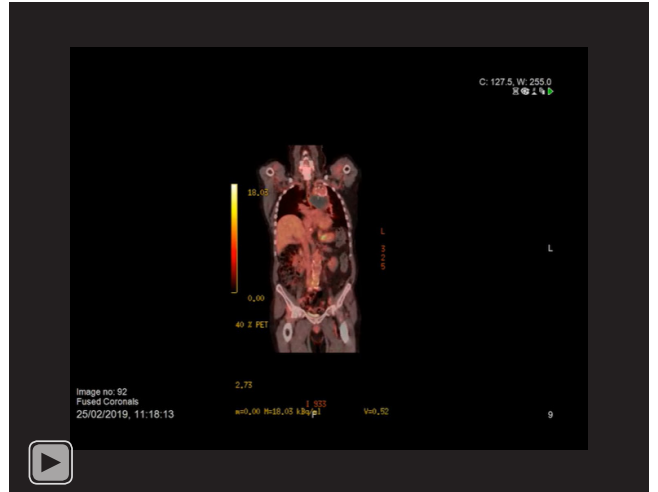

Video 1 Coronal views of the preoperative PET-CT confirming the presence of the endovascular infection by demonstrating intense avidity on the innominate artery and distal aortic arch.

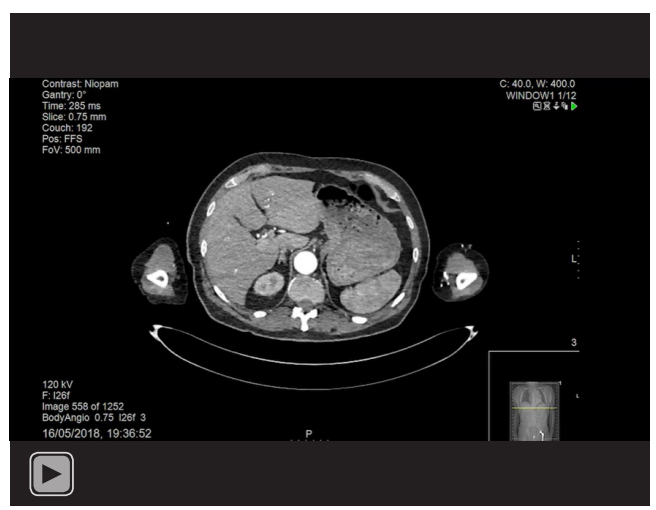

Video 2 Axial views of the preoperative contrasted CT Aorta demonstrating the total arch and descending thoracic aorta endovascular repair with ZenithTM Arch and Alpha distal extension modular stents (Cook) finishing above the visceral segment. Note the residual chronic aneurysm sac within the descending thoracic aorta, without the presence of any endoleaks. Due to the stent graft infection there is a dislocation of the innominate artery stent branch and an expanding aneurysm on the same vessel.

aneurysm sac extending to the distal descending thoracic aorta (Video 2; Figures 2-4).

We studied his cardiovascular function with a myocardial perfusion test that revealed a normal left ventricular function and no signs of ischaemia.

A carotid duplex revealed partially reversed flow on the right carotid artery and fully reversed flow in the right vertebral artery, with an organized thrombus in the right brachial bifurcation.

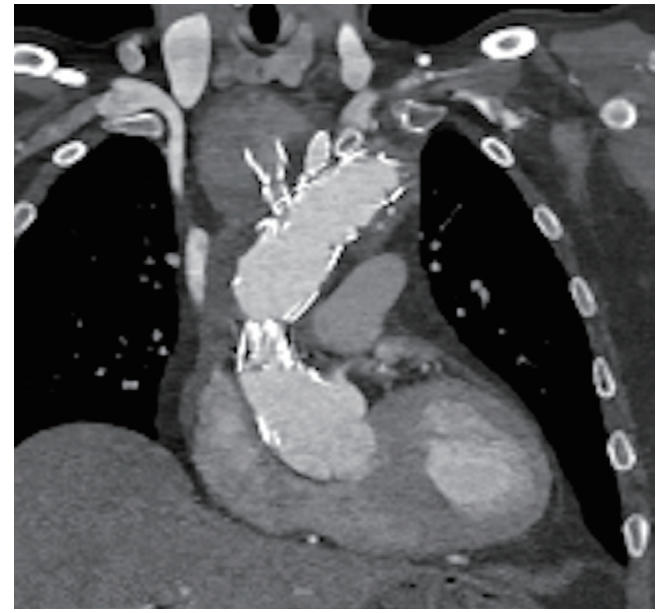

Figure 2 Coronal view of a preoperative contrasted CT showing the presence of the stent extending from the mid ascending aorta into the arch, with two stent branches for the innominate artery and left common carotid artery. The innominate artery stent branch is dislocated due to the infection and there is an expanding aneurysm on the innominate artery.

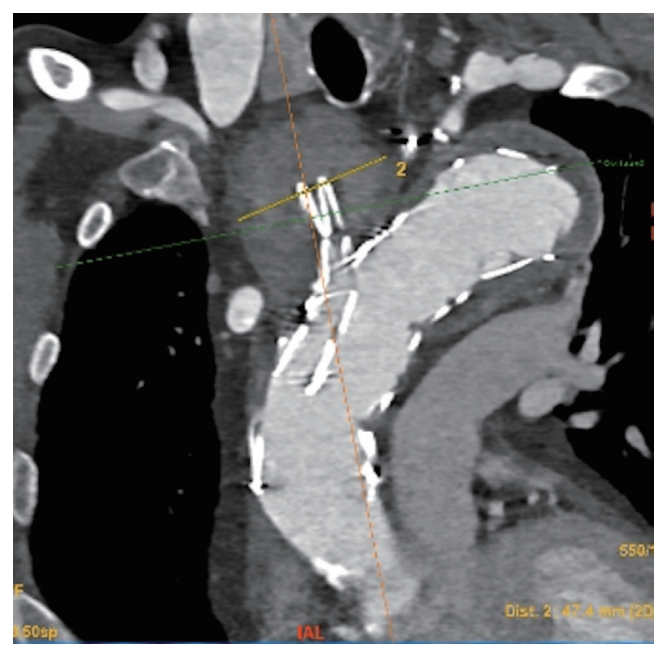

Figure 3 Sagittal view of a preoperative contrasted CT showing the main stent body within the aortic arch and the stent branch into the innominate artery with the accompanying aneurysm.

Lung function tests revealed a mild restrictive respiratory pattern.

Repeated blood cultures were negative, as expected due to the prolong treatment with antibiotics. His white cell blood count was within normal range but $\mathrm{C}$-reactive protein (CRP) levels were elevated at $30 \mathrm{mg} / \mathrm{L}$. 


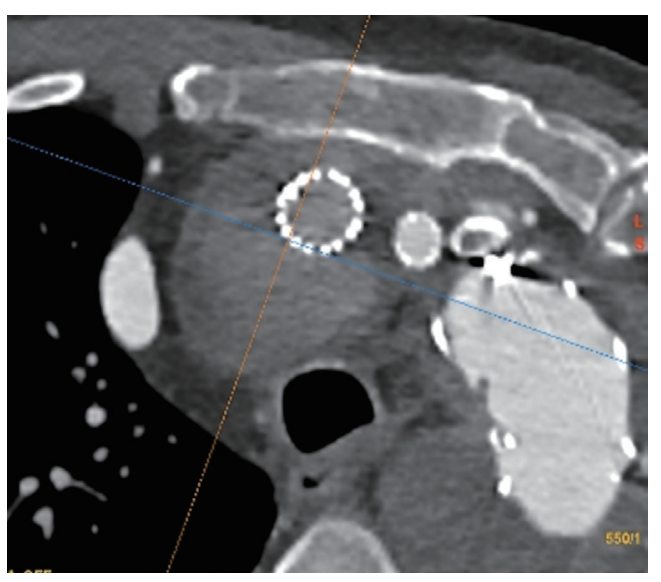

Figure 4 Axial view of a preoperative contrasted CT at the level of the supra-aortic trunks, demonstrating the stent branches in the innominate artery and left common carotid artery, as well as the main stent in the aortic arch and the presence of a large expanding aneurysm in the innominate artery.

\section{Surgical procedure}

Indication for urgent surgical repair was made based on confirmed infection of the endovascular graft and expansion of the innominate artery aneurysm due to dislocation of the stent branch. We planned to remove the infected endovascular graft via median sternotomy with cardiopulmonary bypass, followed by reconstruction of the aortic arch/descending thoracic aorta and supra-aortic vessels with a frozen elephant trunk device using moderate hypothermic circulatory arrest and antegrade cerebral perfusion (Video 3, Figure 1).

Intraoperative monitoring consisted on right radial and right femoral arterial lines, venous central line and nearinfra-red spectrometry (NIRS) for cerebral, paraspinal and lower limb saturations.

The right axillary artery was exposed and after administration of 5,000 units of Heparin, an $8 \mathrm{~mm}$ Dacron graft was anastomosed end to side to the artery in anticipation for antegrade cerebral perfusion delivery.

A median sternotomy was then performed, extending the skin incision cranially into the neck to facilitate access to the supra aortic vessels. After full heparinisation ( $3 \mathrm{mg} / \mathrm{kg})$, cardiopulmonary bypass was established by cannulating the proximal ascending aorta, in an area proximal to the stent, and the right atrium. A vent was inserted in the left ventricle via the right superior pulmonary vein. Myocardial protection was achieved by delivery of cold blood

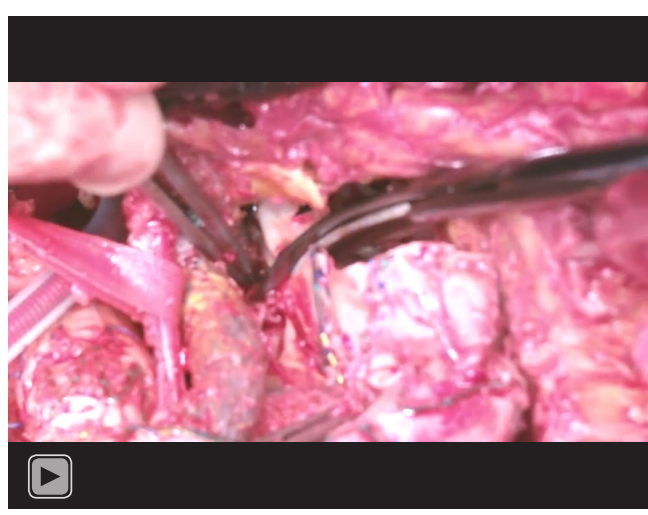

Video 3 Intraoperative video showing the sequence of the operation highlighting the removal of the stent and infected material and further reconstruction of the aortic arch, proximal descending thoracic aorta and supra-aortic trunks using a Thoraflex Plexus $^{\text {TM }}$ (Terumo Aortic, Vascutek) with cardiopulmonary bypass, moderate hypothermic circulatory arrest and antegrade cerebral perfusion.

cardioplegia via antegrade route with a $\mathrm{DLP}^{\circledR}$ antegrade cardioplegia cannula (Medtronic) inserted in the aortic root.

Cooling process started and, in the meantime, full mobilisation of the supra aortic trunks was achieved passing a tape around the innominate and the left common carotid arteries and snaring them before manipulation of the aorta to prevent embolism of infected debris.

At 22 degrees and in Trendelenburg position, the circulation was stopped, and the circulation drained into the venous reservoir.

The proximal ascending aorta was then opened at the level of the proximal end of the stent.

The aorta was then opened longitudinally towards the arch, exposing the whole length of the stent, and reaching a cavity with thrombus that was evacuated. The stent and its two chimneys were mobilized finding extensive amount purulent material between the stent and the aneurysm sac at the level of the distal arch/proximal descending aorta. Antegrade cerebral perfusion was then administered by cannulating the side arm sewn onto the right axillary artery and the left common carotid artery directly from its origin. All the debris and purulent material were evacuated and sent for culture and the stent was cut transversally with scissors as distally as possible (Figure 5). The remaining aneurysmal sac at the aortic arch was then resected and the proximal end of the remaining stent was fixed to the descending thoracic aorta with three interrupted $3 / 0$ pledgeted 


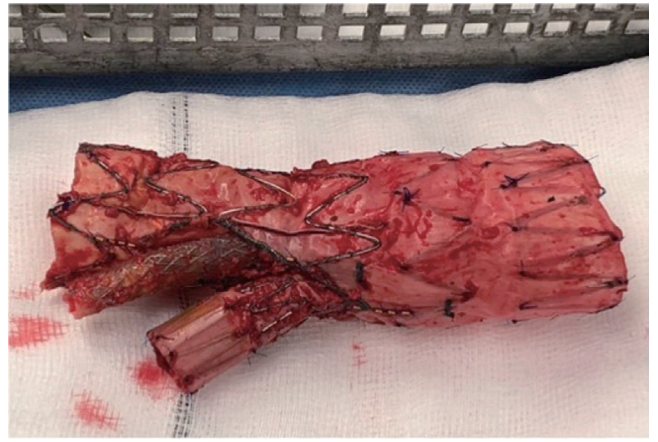

Figure 5 Stent used for the previous endovascular arch repair after surgical removal. Note that the end had to be cut as distal as possible on the descending thoracic aorta as it was not possible to retrieve the whole stent via the median sternotomy.

polypropylene sutures in preparation for deployment of the Thoraflex ${ }^{\mathrm{TM}}$ device. A previously measured, 30/36 $\times 15 \mathrm{~mm}$ Thoraflex Plexus 4 Hybrid $^{\mathrm{TM}}$ Stent Device (Terumo Aortic, Vascutek, Scotland, UK) was deployed antegradely into the descending thoracic aorta and the collar was sutured to the proximal rim of the descending aorta with a buttressed two layered 3/0 polypropylene suture.

Distal circulation was then restored by cannulating the perfusion arm of the Thoraflex ${ }^{\mathrm{TM}}$ prosthesis and cross clamping the graft prior to the side arm origin as well as the origin of the branches for the supra-aortic trunks.

The proximal anastomosis between the Dacron graft and the ascending aorta was then performed using a buttressed double layer $3 / 0$ polypropylene suture technique. After de-airing the heart, the cross clamp was released and rewarming started.

The left common carotid was then anastomosed to the corresponding branch using a continuous $5 / 0$ polypropylene suture. The innominate artery aneurysm was then excised at the level of the bifurcation of the right common carotid and right subclavian arteries. The right common carotid was reimplanted directly onto the corresponding branch of the Thoraflex ${ }^{\mathrm{TM}}$ Plexus, while a separate $8 \mathrm{~mm}$ graft was anastomosed end to end to the right subclavian artery and the other end joint to the remnant branch on the Thoraflex ${ }^{\mathrm{TM}}$ prosthesis.

Once all the anastomoses were completed and the patient warmed $\left(36.7{ }^{\circ} \mathrm{C}\right.$ nasopharyngeal and $35.5^{\circ} \mathrm{C}$ bladder), cardiopulmonary bypass was weaned easily with a moderate inotropic and vasopressor support and paced via biventricular epicardial pacing wires. After haemostasis was achieved, the chest was closed in layer onto two mediastinal

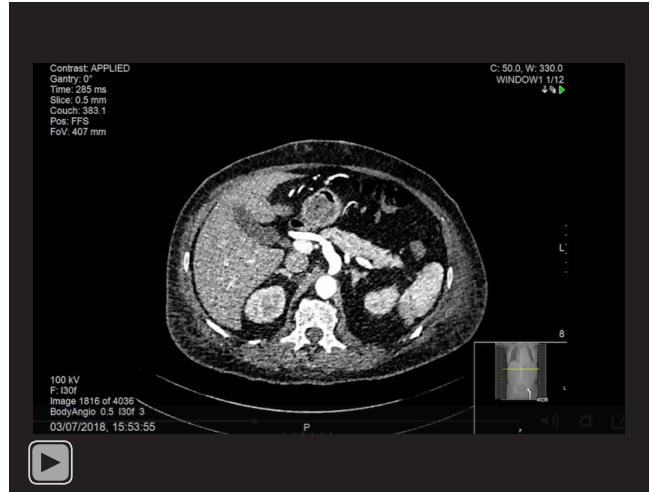

Video 4 Axial views of the postoperative contrasted CT Aorta, demonstrating a satisfactory aortic repair with a Thoraflex ${ }^{\mathrm{TM}}$ Plexus. The stented portion sits inside the previous stent of the descending thoracic aorta. The aortic arch was replaced with individual reimplantation of the right subclavian artery and both right and left common carotid arteries. Previous bypass from left subclavian to left common carotid noted. Note the presence of mild pericardial and bilateral pleural effusions at postoperative day 7 .

and pericardial drains.

\section{Post-operative management}

The patient was transferred to the intensive care unit (ICU), where he completed an uneventful recovery, being extubated on the second postoperative day and discharged to the community hospital, where he completed a six-week course of intravenous antibiotics.

Microbiology culture and sensitivity studies of the purulent material and the removed stent did not reveal the growth of any microorganism. A 16S ribosomal DNA gene polymerase chain reaction (PCR), was also conducted and did not reveal presence of any bacterial pathogens, Consensus between the surgical team and the microbiologists was to continue lifelong empirical antibiotic due to the retained endovascular material in the descending thoracic aorta. He remains on oral Co-trimoxazole and has not developed any symptoms or signs of infection 18 months after the operation (Figure 1).

Immediate postoperative and follow-up CT scans showed a satisfactory aortic repair, with no presence of further collections (Video 4). Due to the presence of a mild pericardial effusion on the CT scan, a transthoracic echocardiogram (TTE) was requested confirming a global effusion, with a maximum diameter of $2.5 \mathrm{~cm}$ laterally, without any haemodynamic repercussion, no respiratory 
variation or right ventricular collapse. This was treated conservatively and had resolved on the repeated TTE at 6 weeks post discharge.

\section{Discussion}

* Endovascular graft infection is a rare but challenging complication, that associates significant morbidity and mortality, and is potentially lethal if untreated.

* Diagnosis is complex, requires a high index of suspicion and it is based on a combination of clinical symptoms, imaging and microbiological investigations (1-4).

* Angio-CT scan is the gold-standard imaging technique, looking for peri-graft gas, fluid collections, soft-tissue attenuation, pseudoaneurysms, discontinuity of the aneurysm wall or fistulous formations. Leukocyte scans and/or Fluorodeoxyglucose (FDG) PET-CT are useful for low-grade infections, showing high avidity within the infected area (1-4).

* There is no standard treatment option and a tailored approach should always be offered. The ultimate goal is to remove the infected graft, but this is not always feasible due to general fitness or co-morbidities. A multidisciplinary team (MDT) discussion is warranted to find a procedure that combines the best morbidity, mortality and durability results (1-4).

* If there is no immediate danger to the patient's life, conservative treatment can be attempted, with antimicrobial therapy guided by sensitivity of the causative organism when available.

Surgery should be attempted as first line in young and fit patients and in those unlikely to be resolved by conservative management (i.e., extensive peri-graft purulence, pseudoaneurysm or suspected aorto-enteric fistula) (1-4).

* Surgical treatment consists on removal of the infected graft followed by revascularization techniques using synthetic, autologous or cryopreserved allografts either with in-situ reconstruction or with extraanatomical bypass techniques (1-4).

* Long-term antibiotic therapy (at least 6 weeks) is always required, with close monitoring of CRP, especially in situations where complete removal of the endovascular material is not possible or associates a greater risk (1-4).

\section{Conclusions}

We report the successful treatment of an endovascular graft infection of a previous total arch and descending aorta endovascular repair. Urgent surgical treatment was warranted by the dislocation of one of the stented branches due to the infection as well as an accompanying expanding aneurysm. Surgical treatment consisted on removal of the infected graft followed by reconstruction of the aortic arch and descending aorta. The patient remains well and without any signs of recurrence, on a long-life oral regime of antibiotics.

\section{Role of team members}

Professor Oo and Benjamin Adams were the surgeons performing the operation and in charge of the postoperative care. Saleem Jahangeer and Ana Lopez-Marco, Aortic Surgery Fellows, wrote the case report, edited the surgical video and prepared the accompanying images and meeting presentation.

\section{Acknowledgments}

This case was presented as a video at the $10^{\text {th }}$ Postgraduate Course on "Surgery of the Thoracic Aorta" in Bologna and awarded one of the Best Video Presentation Prizes and subsequent invitation to contribute a Case Report for the Special Series "Best Video Presentation Prize for the 10th Postgraduate Course on 'Surgery of the Thoracic Aorta' in Bologna".

Funding: None.

\section{Footnote}

Provenance and Peer Review: This article was commissioned by the Guest Editors (Roberto Di Bartolomeo, Davide Pacini and Mohamad Bashir) for the series "Best Video Presentation Prize for the 10th Postgraduate Course on 'Surgery of the Thoracic Aorta' in Bologna" published in Fournal of Visualized Surgery. The article has undergone external peer review.

Reporting Checklist: The authors have completed the CARE reporting checklist. Available at https://jovs.amegroups. com/article/view/10.21037/jovs-20-100/rc

Conflicts of Interest: All authors have completed the ICMJE uniform disclosure form (available at https://jovs. amegroups.com/article/view/10.21037/jovs-20-100/coif). The series "Best Video Presentation Prize for the 10th 
Postgraduate Course on 'Surgery of the Thoracic Aorta' in Bologna" was commissioned by the editorial office without any funding or sponsorship. The authors have no other conflicts of interest to declare.

Ethical Statement: The authors are accountable for all aspects of the work in ensuring that questions related to the accuracy or integrity of any part of the work are appropriately investigated and resolved. Written informed consent was obtained from the patient for video recording and use of the anonymised material for teaching and research purposes. All procedures performed in this study were in accordance with the ethical standards of the institutional and/or national research committee(s) and with the Helsinki Declaration (as revised in 2013).

Open Access Statement: This is an Open Access article distributed in accordance with the Creative Commons Attribution-NonCommercial-NoDerivs 4.0 International License (CC BY-NC-ND 4.0), which permits the noncommercial replication and distribution of the article with the strict proviso that no changes or edits are made and the original work is properly cited (including links to both the formal publication through the relevant DOI and the license). See: https://creativecommons.org/licenses/by-nc-nd/4.0/.

\section{References}

1. Setacci C, Chisci E, Setacci F, et al. How to diagnose and manage infected endografts after endovascular aneurysm repair. Aorta (Stamford) 2014;2:255-64.

2. Wilson WR, Bower TC, Creager MA, et al. Vascular graft infection, mycotic aneurysms and endovascular infections. A scientific statement from the American Heart Association. Circulation 2016;134:412-60.

3. Lyons OT, Baguneid M, Barwick TD, et al. Diagnosis of aortic graft infection: a case definition by the Management of Aortic Graft Infection Collaboration (MAGIC). Eur J Vasc Endovasc Surg 2016;52:758-63.

4. Schaefers JF, Donas KP, Panuccio G, et al. Outcomes of surgical explantation of infected aortic grafts after endovascular and open abdominal aneurysm repair. Eur J Vasc Endovasc Surg 2019;57:130-6. doi: 10.21037/jovs-20-100

Cite this article as: Lopez-Marco A, Jahangeer S, Adams B, Oo AY. Arch replacement following endovascular arch repair for an infected stent: case report. J Vis Surg 2022;8:9. 